\title{
The Steiner $k$-Wiener index of graphs with given minimum degree
}

\author{
Peter Dankelmann (University of Johannesburg)
}

May 15, 2018

\begin{abstract}
Let $G$ be a connected graph. The Steiner distance $d(S)$ of a set $S$ of vertices is the minimum size of a connected subgraph of $G$ containing all vertices of $S$. For $k \in \mathbb{N}$, the Steiner $k$-Wiener index $S W_{k}(G)$ is defined as $\sum_{S} d(S)$, where the sum is over all $k$-element subsets of the vertex set of $G$. The average Steiner $k$-distance $\mu_{k}(G)$ of $G$ is defined as $\left(\begin{array}{l}n \\ k\end{array}\right)^{-1} S W_{k}(G)$.

In this paper we prove upper bounds on the Steiner Wiener index and the average Steiner distance of graphs with given order $n$ and minimum degree $\delta$. Specifically we show that $S W_{k}(G) \leq \frac{k-1}{k+1} \frac{3 n}{\delta+1}\left(\begin{array}{l}n \\ k\end{array}\right)+O\left(n^{k}\right)$, and that $\mu_{k}(G) \leq$ $\frac{k-1}{k+1} \frac{3 n}{\delta+1}+O(1)$. We improve this bound for triangle-free graphs to $S W_{k}(G) \leq$ $\frac{k-1}{k+1} \frac{2 n}{\delta}\left(\begin{array}{l}n \\ k\end{array}\right)+O\left(n^{k}\right)$, and $\mu_{k}(G) \leq \frac{k-1}{k+1} \frac{2 n}{\delta}+O(1)$. All bounds are best possible.
\end{abstract}

Keywords: Steiner Wiener index; average Steiner distance; Wiener index; average distance; Steiner distance; transmission

MSC-class: 05C12 (primary) 92E10 (secondary)

\section{Introduction}

The Wiener index $W(G)$ of a connected graph $G$ is defined as the sum of the distances between all unordered pairs of vertices, i.e.,

$$
W(G)=\sum_{\{u, v\} \subseteq V(G)} d(u, v),
$$

where $V(G)$ is the vertex set of $G$, and $d(u, v)$ is the usual distance, i.e., the minimum length of a path from $u$ to $v$. First studied by the chemist Wiener [33 as an indicator for the boiling point of certain alkanes, the Wiener index has become one of the most important topological indices in chemical graph theory. For its many applications see, for example, the survey [30]. Since its inception, the Wiener index has attracted much interest in the graph theory literature under different names, 
such as transmission, defined as the sum of the distances between all ordered pairs of vertices, and total distance. The Wiener index is closely related to the average distance $\mu(G)$, also known as mean distance, defined as the average of all distances between two vertices of $G$, i.e.,

$$
\mu(G)=\left(\begin{array}{l}
n \\
2
\end{array}\right)^{-1} \sum_{\{u, v\} \subseteq V(G)} d(u, v),
$$

where $n$ is the order of $G$. Hence $W(G)=\left(\begin{array}{l}n \\ 2\end{array}\right) \mu(G)$.

The Steiner distance $d_{G}(S)$ of a set $S$ of vertices in a connected graph $G$ is defined as the minimum size of a connected subgraph of $G$ containing all vertices of $S$. This concept was introduced by Chartrand, Oellermann, Tian and Zou [5] in order to generalise the notion of distance between two vertices in a graph to an arbitrary number of vertices. For $k \in \mathbb{N}$, the maximum value of $d_{G}(S)$, taken over all $k$-sets of vertices of $G$, is known as the $k$-Steiner diameter or $k$-diameter of $G$. For results on the Steiner diameter see, for example, [1, 2, 3, 7] and the survey paper [25].

This paper is concerned with the Steiner $k$-Wiener index, which generalises the Wiener index by combining the notions of Steiner distance and Wiener index. For $k \in \mathbb{N}$, the Steiner $k$-Wiener index $S W_{k}(G)$ of a connected graph $G$ is defined as the sum of the Steiner distances of all $k$-sets of vertices, i.e.,

$$
S W_{k}(G)=\sum_{S \subseteq V(G),|S|=k} d_{G}(S)
$$

It generalises the Wiener index since clearly $S W_{2}(G)=W(G)$. The Steiner $k$ Wiener index was introduced by Li, Mao and Gutman [21]. A closely related graph parameter, the average Steiner $k$-distance of $G$, denoted by $\mu_{k}(G)$ and defined as the average of the Steiner distances of all $k$-element subsets of $V(G)$, was introduced in [6] and further investigated in [8]. In the same way in which the Steiner $k$-Wiener index generalises the Wiender index, the average $k$-distance generalises the average distance since $\mu_{2}(G)=\mu(G)$.

Several results on the Wiener index are known to hold also for the Steiner $k$ Wiener index. The observation that the Wiener index of a graph of order $n$ is at least $\left(\begin{array}{l}n \\ 2\end{array}\right)$ easily extends to the Steiner $k$-Wiener index: since the Steiner distance of a set of $k$ vertices is at least $k-1$, we obtain the lower bound $S W_{k}(G) \geq(k-1)\left(\begin{array}{l}n \\ k\end{array}\right)$. Doyle and Graver [14], Entringer, Jackson and Snyder [15] and Lovász [23] independently observed that

$$
W(G) \leq \frac{n+1}{3}\left(\begin{array}{l}
n \\
2
\end{array}\right)
$$

with equality if and only if $G$ is a path. Dankelmann, Oellermann and Swart [6] and Li, Mao, Gutman [21] showed that this result extends to the Steiner $k$-Wiener index. 
Theorem 1. 6], 21] Let $G$ be a graph of order $n$. Then

$$
S W_{k}(G) \leq \frac{(k-1)(n+1)}{k+1}\left(\begin{array}{l}
n \\
k
\end{array}\right)
$$

Equality holds if $G$ is a path.

It was observed by Plesník [29] that among all trees of given order the star minmimises the Wiener index. It was shown by Dankelmann, Oellermann and Swart [6], and by Li, Mao and Gutman [21 that the same statement holds also for the Steiner $k$-Wiener index. Plesník [29] also showed that among all trees of order $n$ and diameter $d$, the Wiener index is minimised by the tree obtained from a path on $d+1$ vertices by attaching $n-d-1$ vertices to a centre vertex of the path. This result was shown in [24] to hold for the Steiner $k$-Wiener index. Nordhaus-Gaddum type results on the Wiener index [35] were generalised to the Steiner $k$-Wiener index [27], and so were results on the inverse Wiener problem, i.e., the question which numbers are the Wiener index of some graph [31, 32, 22], as well as results on product graphs (see [34, 26]).

The bound on the Wiener index (1) has been improved for graphs with various given properties. For example for 2-connected graphs (i.e., connected graphs in which removing a vertex does not disconnect the graph), Plesník [29] showed that

$$
W(G) \leq \frac{n}{2}\left\lfloor\frac{n^{2}}{4}\right\rfloor
$$

with equality if and only if $G$ is a cycle. An extension of this result was given in [7], where it was shown that the cycle maximises, among all 2-connected graphs of given order, the Steiner $k$-Wiener index for every integer $k$ with $2 \leq k \leq n$. Inequality (2) was strengthened for graphs with given order and connectivity [12 and edgeconnectivity [10, 11], but no bounds on the Steiner $k$-Wiener index of graphs of given connectivity or edge-connectivity appear to be known.

The bound in (11) has also been improved for graphs of given minimum degree. The computer program GRAFFITI conjectured that the average distance of a graph of order $n$ and minimum degree $\delta$ is not more than $\frac{n}{\delta}$. Kouider and Winkler [20] proved the following result which is asymptotically sharp.

Theorem 2. 20] Let $G$ be a connected graph of order $n$ and minimum degree $\delta$. Then

$$
W(G) \leq\left(\frac{n}{\delta+1}+2\right)\left(\begin{array}{l}
n \\
2
\end{array}\right) .
$$

Although Theorem 2 is asymptotically stronger than the above-mentioned GRAFFITI conjecture, it does not actually imply it. The GRAFFITI conjecture, was proved later by Beezer, Riegsecker and Smith [4]. Kouider and Winkler's result was extended in two ways by Dankelmann and Entringer [9] by showing that the above bound holds not only for $G$ but for some spanning tree of $G$, and further by improving this bound by a factor of about $\frac{2}{3}$ for triangle-free graphs. It is the aim 
of this paper to show that these bounds on the Wiener index of graphs in terms of order and minimum degree extend to the Steiner $k$-Wiener index and the average Steiner $k$-distance.

We note that there are several recent bounds on the Wiener index, for example in terms of diameter [28], radius [13], and other results [17, 18, 19] on the Wiener index which have not (yet) been shown to hold for the Steiner $k$-Wiener index.

\section{Notation}

The notation we use is as follows. By $G$ we always denote a finite, simple, connected graph on $n(G)$ vertices with vertex set $V(G)$. For a vertex $v$ of $G, N_{G}(v)$ is the neighbourhood of $v$, i.e., the set of vertices adjacent to $v$, and $N_{G}[v]$ is the closed neighbourhood of $v$, i.e., the set $N_{G}(v) \cup\{v\}$. For $A \subseteq V(G)$ we define $N[A]=$

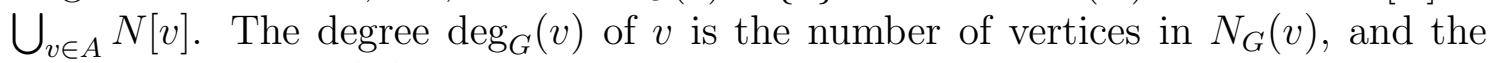
minimum degree $\delta(G)$ of $G$ is the smallest of the degrees of the vertices of $G$. If $U \subseteq E(G)$, then $V(U)$ is the set of vertices of $G$ incident with at least one edge in $U$, and $G[U]$, the subgraph induced by $U$, is the subgraph whose vertex set is $V(U)$ and whose edge set is $U$.

The distance between two vertices $u$ and $v$, i.e., the minimum length of a $(u, v)$ path, is denoted by $d_{G}(u, v)$. If $S$ is a non-empty subset of the vertex set of $G$, then the Steiner distance of $S, d_{G}(S)$, is the minimum size of a connected subgraph of $G$ containing the vertices of $S$. The distance between a vertex $v$ and a set $A$ of vertices of $G$ is defined as $\min _{w \in A} d_{G}(v, w)$. If the graph is understood from the context, then we sometimes omit the argument or subscript $G$.

By $K_{n}$ we mean the complete graph on $n$ vertices, and $n K_{1}$ denotes the edgeless graph on $n$ vertices. For disjoint graphs $G_{1}, G_{2}, \ldots, G_{k}$ the sequential sum $G_{1}+$ $G_{2}+\cdots+G_{k}$ is the graph obtained from the union of $G_{1}, G_{2}, \ldots, G_{k}$ by joining every vertex of $G_{i}$ to every vertex of $G_{i+1}$ for $i=1,2, \ldots, k-1$. The line graph of a graph $G$ is the graph whose vertices are the edges of $G$, with two vertices of the line graph being adjacent if the corresponding edges of $G$ have a vertex in common.

\section{$3 \quad$ Weighted Steiner $k$-Wiener index}

In this section we introduce the weighted Steiner $k$-Wiener index, which generalises the weighted Wiener index. The main result of this section, Lemma 2, is a common generalisation of Theorem 1 and a bound on the weighted Wiener index given in [9]. For the proof of Lemma 2 we require the following notation.

Definition 1. Given a set $X$ and $c$ a weight function $c: X \rightarrow \mathbb{N}_{0}$.

(a) For $Y \subseteq X$ we define $c(Y)$ as $\sum_{y \in Y} c(y)$.

(b) Define $X_{c}$ to be the set obtained from $X$ by replacing every $x \in X$ with $c(x) \geq 1$ by elements $x^{1}, x^{2}, \ldots, x^{c(x)}$ and deleting all $y \in X$ for which $c(y)=0$. If $c(x)>0$ then we refer to $x$ as the original, and to $x^{1}, x^{2}, \ldots, x^{c(x)}$ as the copies of $x$. 
(c) Given a set $Y \subseteq X_{c}$, the original of $Y$ is the set $Y^{*} \subset X$ whose elements are exactly those $x \in X$ of which $Y$ contains at least one copy.

(d) If $X$ is the vertex set of a graph $G$, then for $Y \subseteq X_{c}$ we define $d(Y)$ as $d_{G}\left(Y^{*}\right)$.

It is easy to see that $\left|X_{c}\right|=c(X)$. With the above definition we can now introduce the weighted Steiner $k$-Wiener index of a graph.

Definition 2. Let $G$ be a connected graph with vertex set $V$ and $c: V \rightarrow \mathbb{N}_{0}$ a weight function. The Steiner $k$-Wiener index of $G$ with respect to $c$ is defined by

$$
S W_{k}(G, c)=\sum_{S \subseteq V_{c},|S|=k} d_{G}(S)
$$

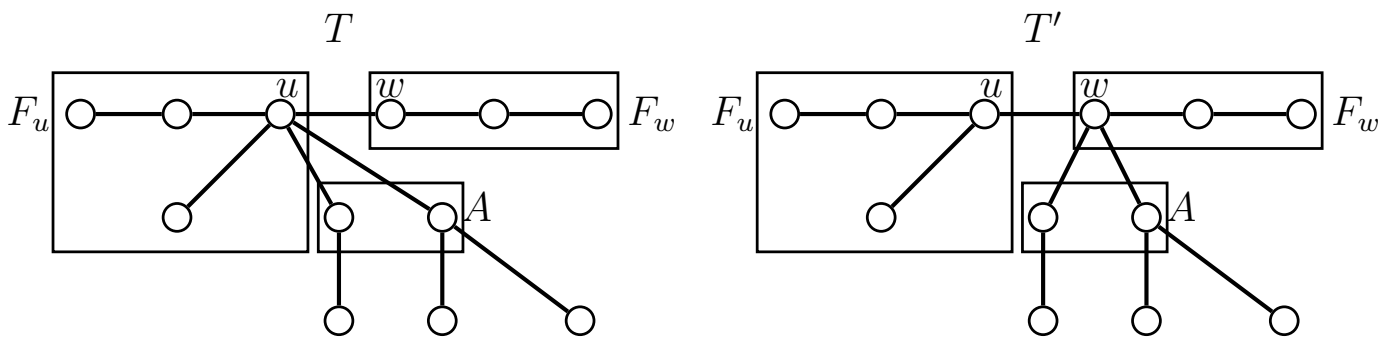

Figure 1: The trees $T$ and $T^{\prime}$ in Lemma 1.

Lemma 1. Given a tree $T$ with a weight function $c: V(T) \rightarrow \mathbb{N}_{0}$. Let $u, w$ be two adjacent vertices of $T$. Let $A \subseteq N(u)-\{w\}$ be a nonempty set. Let $F$ be the component of $T-\{u a \mid a \in A\}$ containing $u$. Let $F_{u}$ and $F_{w}$ be the components of $F-u w$ containing $u$ and $w$, and $U$ and $W$ their vertex sets, respectively. Let $T^{\prime}$ be the tree $T-\{u a \mid a \in A\}+\{w a \mid a \in A\}$. If $c(U)>c(W)$, then

$$
S W_{k}(T, c)<S W_{k}\left(T^{\prime}, c\right)
$$

Proof: For a sketch showing $T$ and $T^{\prime}$ see Figure 1. Let $V$ be the common vertex set of $T$ and $T^{\prime}$, and let $S$ be a $k$-element subset of $V_{c}$. If $S^{*} \cap(U \cup W)=\emptyset$, then clearly $d_{T^{\prime}}(S)=d_{T}(S)$. Similarly, if $S^{*}$ contains elements of both, $U$ and $W$, then $d_{T^{\prime}}(S)=d_{T}(S)$. Hence $d_{T^{\prime}}(S) \neq d_{T}(S)$ only if $S^{*}-(U \cup W) \neq \emptyset$ and exactly one of $S^{*} \cap U$ and $S^{*} \cap W$ is nonempty. Denoting $V(T)-(U \cup W)$ by $X$ we obtain

$$
\begin{aligned}
S W_{k}\left(T^{\prime}, c\right)-S W_{k}(T, c)=\sum_{S \subseteq V_{c},|S|=k}\left(d_{T^{\prime}}(S)-d_{T}(S)\right) & \left(d_{T^{\prime}}(S)-d_{T}(S)\right)+\sum_{S: S^{*} \cap X \neq \emptyset, S^{*} \cap W \neq \emptyset, S^{*} \cap U=\emptyset} \sum_{S: S^{*} \cap X \neq \emptyset, S^{*} \cap U \neq \emptyset, S^{*} \cap W=\emptyset}\left(d_{T^{\prime}}(S)-d_{T}(S)\right) .
\end{aligned}
$$

If $S \subseteq V_{c}$ satisfies $S^{*} \cap X \neq \emptyset, S^{*} \cap U \neq \emptyset$ and $S^{*} \cap W=\emptyset$, then $d_{T^{\prime}}(S)-d_{T}(S)=1$. Similarly, if $S \subseteq V_{c}$ satisfies $S^{*} \cap X \neq \emptyset, S^{*} \cap W \neq \emptyset$ and $S^{*} \cap U=\emptyset$, then 
$d_{T^{\prime}}(S)-d_{T}(S)=-1$. Hence

$$
\begin{aligned}
S W_{k}\left(T^{\prime}, c\right)-S W_{k}(T, c)= & \left|\left\{S \subseteq V_{c}|| S \mid=k, S^{*} \cap X \neq \emptyset, S^{*} \cap U \neq \emptyset, S^{*} \cap W=\emptyset\right\}\right| \\
& -\left|\left\{S \subseteq V_{c}|| S \mid=k, S^{*} \cap X \neq \emptyset, S^{*} \cap W \neq \emptyset, S^{*} \cap U=\emptyset\right\}\right| \\
= & \sum_{i=1}^{k-1}\left(\begin{array}{c}
c(U) \\
i
\end{array}\right)\left(\begin{array}{c}
c(X) \\
k-i
\end{array}\right)-\sum_{i=1}^{k-1}\left(\begin{array}{c}
c(W) \\
i
\end{array}\right)\left(\begin{array}{c}
c(X) \\
k-i
\end{array}\right) \\
= & \sum_{i=1}^{k-1}\left(\begin{array}{c}
c(X) \\
k-i
\end{array}\right)\left[\left(\begin{array}{c}
c(U) \\
i
\end{array}\right)-\left(\begin{array}{c}
c(W) \\
i
\end{array}\right)\right] \\
> & 0,
\end{aligned}
$$

where the last inequality holds since $c(U)>c(W)$. Hence the lemma follows.

The following lemma is central to our proof of the main results of this paper. For trees it is a generalisation of inequality (11), which is the special case $k=2$ and $C=1$.

Lemma 2. Let $T$ be a tree with vertex set $V$. Let $c: V \rightarrow \mathbb{N}_{0}$ be a weight function on the vertex set of $T$ such that $c(v) \geq C$ for every vertex $v \in V$. Let $N$ be the total weight of the vertices of $T$. If $C \geq 1$, then

$$
S W_{k}(T, c) \leq \frac{k-1}{k+1} \frac{N+1}{C}\left(\begin{array}{l}
N \\
k
\end{array}\right)+\frac{C-1}{C}\left(\begin{array}{l}
N \\
k
\end{array}\right),
$$

Proof: Let $N$ and $C$ be fixed. We may assume that among all trees and weight functions satisfying the hypothesis of the lemma, $T$ and $c$ are chosen such that $S W_{k}(T, c)$ is maximum.

Claim 1: $T$ is a path.

Suppose to the contrary that $T$ is not a path. Then $T$ contains a vertex $u$ of degree at least three. Let $V_{1}, V_{2}, \ldots, V_{r}$ be the vertex sets of the components of $T-u$ and let $w_{i}$ the neighbour of $u$ in $V_{i}$ for $i=1,2, \ldots, r$. We may assume that $c\left(V_{1}\right) \geq c\left(V_{2}\right) \geq \ldots \geq c\left(V_{r}\right)$. Let $A=\left\{w_{1}, w_{2}, \ldots, w_{r-2}\right\}$. Define the tree $T^{\prime}$ by

$$
T^{\prime}=T-\{u a \mid a \in A\}+\left\{w_{r} a \mid a \in A\right\} .
$$

Then the hypothesis of Lemma 1 is satisfied with vertices $u$ and $w_{r}$ corresponding to vertices $u$ and $w$ in Lemma 1, the subtree $T\left[V_{r-1} \cup V_{r} \cup\{u\}\right]$ corresponding to $F$ in Lemma 1, and the sets $\{u\} \cup V_{r-1}$ and $V_{r}$ corresponding to $U$ and $W$. We have $c\left(\{u\} \cup V_{r-1}\right)>c\left(V_{r-1}\right) \geq c\left(V_{r}\right)$. Hence the hypothesis of Lemma 1 is satisfied, and if follows that

$$
S W_{k}\left(T^{\prime}, c\right)>S W_{k}(T, c) .
$$

which is a contradiction to the maximality of $S W_{k}(T, c)$. Hence $T$ is a tree with no vertex of degree greater than two, so $T$ is a path. This proves Claim 1. 
From now on we assume that $T$ is a path with vertices $v_{1}, v_{2}, \ldots, v_{n}$ in this order. We now define an auxiliary graph $P$ on the vertex set $V_{c}$. Define the path $P$ by

$$
P=v_{1}^{1}, v_{1}^{2}, \ldots, v_{1}^{c\left(v_{1}\right)}, v_{2}^{1}, v_{2}^{2}, \ldots, v_{2}^{c\left(v_{2}\right)}, \ldots, v_{n}^{1}, v_{n}^{2}, \ldots, v_{n}^{c\left(v_{n}\right)} .
$$

Claim 2: For any two vertices, $x, y$ of $P$ we have $d_{T}(x, y) \leq \frac{d_{P}(x, y)-1}{C}+1$.

Let $x=v_{i}^{r}$ and $y=v_{j}^{s}$. The statement clearly holds if $i=j$, hence we assume, without loss of generality, that $i<j$. Then, since $c\left(v_{i}\right), c\left(v_{i+1}\right), \ldots, c\left(v_{j}\right) \geq C$ we obtain

$$
\begin{aligned}
d_{P}\left(v_{i}^{r}, v_{j}^{s}\right) & \geq d_{P}\left(v_{i}^{c\left(v_{i}\right)}, v_{j}^{1}\right) \\
& =c\left(v_{i+1}\right)+c\left(v_{i+2}\right)+\cdot+c\left(v_{j-1}\right)+1 \\
& \geq C(j-i-1)+1 \\
& =C\left(d_{T}\left(v_{i}, v_{j}\right)-1\right)+1,
\end{aligned}
$$

and by rearranging we get Claim 2 .

Claim 3: If $S \subseteq V_{c},|S| \geq 2$, then $d_{T}(S) \leq \frac{d_{P}(S)-1}{C}+1$.

Since $T$ and $P$ are paths, we have $d_{P}(S)=\max _{x, y \in S} d_{P}(x, y)$ and $d_{T}(S)=\max _{x, y \in S} d_{T}(x, y)$. Hence, by Claim 2,

$$
d_{T}(S)=\max _{x, y \in S} d_{T}(x, y) \leq \frac{\max _{x, y \in S} d_{P}(x, y)-1}{C}+1=\frac{d_{P}(S)-1}{C}+1,
$$

which proves Claim 3.

We now complete the proof. By Claim 3,

$S W_{k}(T, c)=\sum_{S \subseteq V_{c},|S|=k} d_{T}(S) \leq \sum_{S \subseteq V_{c},|S|=k}\left(\frac{d_{P}(S)}{C}+\frac{C-1}{C}\right)=\frac{1}{C} S W_{k}(P)+\frac{C-1}{C}\left(\begin{array}{c}N \\ k\end{array}\right)$.

By (11) we have $S W_{k}(P)=\frac{(k-1)(N+1)}{k+1}\left(\begin{array}{l}N \\ k\end{array}\right)$. Substituting this into the previous inequality we get

$$
S W_{k}(T, c) \leq \frac{1}{C} \frac{(k-1)(N+1)}{k+1}\left(\begin{array}{c}
N \\
k
\end{array}\right)+\frac{C-1}{C}\left(\begin{array}{c}
N \\
k
\end{array}\right)
$$

as desired.

\section{A bound in terms of order and minimum degree}

In the proof of the following theorem we employ a refinement of a method that was developed in [9] to prove an upper bound on the average distance. For a vertex $v$ of $G$ denote by $T(a)$ the subtree of $G$ whose vertex set is $N[v]$, and whose edges are the edges joining $v$ to its neighbours in $G$. 
Theorem 3. Let $G$ be a connected graph of order $n$ with minimum degree $\delta$. Then $G$ contains a spanning tree $T$ with

$$
S W_{k}(T) \leq \frac{k-1}{k+1} \frac{3(n+1)}{\delta+1}\left(\begin{array}{l}
n \\
k
\end{array}\right)+\left(\frac{3 \delta}{\delta+1}+2 k\right)\left(\begin{array}{l}
n \\
k
\end{array}\right),
$$

Proof: The strategy of the proof is as follows. We simultaneously construct a maximum packing $A$ in $G$ and a subtree $T_{0}$ of $G$, which we extend to a spanning tree $T$ of $G$. We then show that the bound of the theorem holds for $T$.

To construct a packing of $G$ start by choosing a vertex $a_{1}$ and letting $A_{1}=\left\{a_{1}\right\}$ and $T_{1}=T\left(a_{1}\right)$. Let $a_{2}$ be a vertex at distance exactly 3 from $A_{1}$, if one exists. Then there exists an edge $e_{2}$ joining some vertex of $T_{1}$ to some vertex of $T\left(a_{2}\right)$. Let $A_{2}=A_{1} \cup\left\{a_{2}\right\}$ and let $T_{2}$ be the tree obtained from $T_{1} \cup T\left(a_{2}\right)$ by adding the edge $e_{2}$. Let $a_{3}$ be a vertex at distance exactly 3 from $A_{2}$, if one exists. Then there exists an edge $e_{3}$ joining some vertex of $T_{2}$ to some vertex of $T\left(a_{3}\right)$. Let $A_{3}=A_{2} \cup\left\{a_{3}\right\}$ and let $T_{3}$ be the graph obtained from $T_{2} \cup T\left(a_{3}\right)$ by adding the edge $e_{3}$. Generally, given $A_{i}$ and $T_{i}$, we choose a vertex $a_{i+1}$ at distance exactly 3 from $A_{i}$, if one exists, let $e_{i+1}$ be an edge joining a vertex in $T_{i}$ to a vertex in $T\left(a_{i+1}\right)$, let $A_{i+1}=A_{i} \cup\left\{a_{i+1}\right\}$, and let $T_{i+1}$ be the tree obtained from the disjoint union of $T_{i}$ and $T\left(a_{i+1}\right)$ by adding the edge $e_{i+1}$. Repeat this step until, after $s$ steps say, all vertices are at distance at most 2 from $A_{s}$. Let $T_{0}:=T_{s}$ and $A:=A_{s}$. Then $A$ is a maximal packing and every vertex of $G$ is within distance at most 2 in $G$ from $A$, and thus adjacent to some vertex in $T_{0}$. Joining every vertex not in $T_{0}$ to a neighbour in $T_{0}$ yields a spanning tree $T$ of $G$.

For every vertex $v$ of $T$ choose a nearest vertex $a_{v} \in A$, and a shortest $\left(v, a_{v}\right)$ path $P_{v}$ in $T$. Then $P_{v}$ has at most two edges. For $a \in A$ let $c(a)$ be the number of vertices $v$ of $G$ with $a_{v}=a$. Since $A$ is a packing, for every $a \in A$ all vertices in $N[a]$ have $a$ as their nearest vertex in $A$, and so

$$
c(a) \geq \delta+1 \text { for all } a \in A,
$$

We now show that the difference $S W_{k}(T)-S W_{k}(T, c)$ is at most $O\left(n^{k}\right)$. More specifically, we show that

$$
S W_{k}(T) \leq S W_{k}(T, c)+2 k\left(\begin{array}{l}
n \\
k
\end{array}\right)
$$

Let $c_{1}$ be the weight function that assigns a weight of 1 to each vertex of $T$. Clearly, $S W_{k}(T)=S W_{k}\left(T, c_{1}\right)$. Then $c$ is obtained by moving weight units from $v$ to $a_{v}$ for all vertices $v \in V$. Hence $V_{c}$ is obtained from $V_{c_{1}}$ by moving the copy $v^{1} \in V_{c_{1}}$ of vertex $v$ to vertex $a_{v}$ and making it a copy of $a_{v}$. Hence there is a bijection $f$ between the $k$-subsets of $V_{c_{1}}$ and the $k$-subsets of $V_{c}$, mapping every $k$-set $S_{1} \subset V_{c_{1}}$ to a $k$-set $f(S) \subseteq V_{c}$ by replacing copies (with respect to $c_{1}$ ) of a vertex $v$ by copies of the vertex $a_{v}$ (with respect to $c$ ). If $T_{S}$ is a Steiner tree for a $k$-set $S \subseteq V_{c_{1}}$, then by adding or deleting suitable edges that are in $\bigcup_{v \in S *} E\left(P_{v}\right)$, we obtain a subtree of $T$ containing all vertices in $f(S)^{*}$. Since $\left|\bigcup_{v \in S *} E\left(P_{v}\right)\right| \leq 2 k$, we conclude that

$$
d_{T}(S) \leq d_{T}(f(S))+2 k .
$$


Summing over all $k$-subsets $S \subseteq V_{c_{1}}$ yields (44).

We proceed to bound $S W_{k}(T, c)$. Since by the construction of $T_{0}$ and $T$ every vertex $a_{i}, i>1$, is at distance exactly 3 in $T$ from some vertex $a_{j}$ with $j<i$, it follows that in $T^{3}[A]$ there exists a path from $a_{i}$ to $a_{1}$ for every $i>1$. Hence

$$
T^{3}[A] \text { is connected. }
$$

Let $H=T^{3}[A]$. Since $d_{T}\left(a_{i}, a_{j}\right) \leq 3 d_{H}\left(a_{i}, a_{j}\right)$ for all $a_{i}, a_{j} \in A$, and since the weight of $c$ is concentrated in the vertices of $A$, we have

$$
S W_{k}(T, c) \leq 3 S W_{k}\left(H, c^{\prime}\right)
$$

where $c^{\prime}$ is the restriction of $c$ to $A$. By (3),$c^{\prime}\left(a_{i}\right) \geq \delta+1$ for all $a_{i} \in A$. Moreover, the total weight of $c^{\prime}$ is $n$, i.e., $N=n$. Hence, by Lemma 2

$$
S W_{k}\left(H, c^{\prime}\right) \leq \frac{k-1}{k+1} \frac{n+1}{\delta+1}\left(\begin{array}{l}
n \\
k
\end{array}\right)+\frac{\delta}{\delta+1}\left(\begin{array}{l}
n \\
k
\end{array}\right) .
$$

Applying (44), (6) and (17) we obtain

$$
S W_{k}(T) \leq \frac{k-1}{k+1} \frac{3(n+1)}{\delta+1}\left(\begin{array}{l}
n \\
k
\end{array}\right)+\left(\frac{3 \delta}{\delta+1}+2 k\right)\left(\begin{array}{l}
n \\
k
\end{array}\right),
$$

as desired.

Corollary 1. Let $G$ be a connected graph of order $n$ with minimum degree $\delta$. Then

$$
S W_{k}(G) \leq \frac{k-1}{k+1} \frac{3(n+1)}{\delta+1}\left(\begin{array}{l}
n \\
k
\end{array}\right)+\left(\frac{3 \delta}{\delta+1}+2 k\right)\left(\begin{array}{l}
n \\
k
\end{array}\right)
$$

and thus

$$
\mu_{k}(T) \leq \frac{k-1}{k+1} \frac{3(n+1)}{\delta+1}+\frac{3 \delta}{\delta+1}+2 k .
$$

Example 1. We now construct an example to show that the bound on the Steiner $k$-Wiener index in Corollary 1 is best possible apart from a term $O\left(n^{k}\right)$, and that the bound on the average Steiner $k$-distance is best possible apart from an additive constant. We only construct examples for the case that $\delta+1$ is a multiple of 3 , but it is not difficult to modify this construction for all values of $\delta$. For $d \in \mathbb{N}$ define the graph $G_{d, \delta}$ by

$$
G_{d, \delta}=K_{\delta}+K_{(\delta+1) / 3}+K_{(\delta+1) / 3}+\cdots+K_{(\delta+1) / 3}+K_{(\delta+1) / 3}+K_{\delta},
$$

where the term $K_{(\delta+1) / 3}$ appears $d-1$ times. Clearly, $n\left(G_{d, \delta}\right)=\frac{d+5}{3}(\delta+1)-2$, and $\operatorname{diam}\left(G_{d, \delta}\right)=d$. Hence for large $d$ and constant $\delta$ we have $n=d \frac{\delta+1}{3}+O(1)$. We now bound the Steiner $k$-Wiener index from below. For $i=1,2, \ldots, d-1$ let $V_{i}$ be the set of vertices of the $i$-th copy of $K_{(\delta+1) / 3}$, and let $V_{0}$ and $V_{d}$ be subsets of cardinality $(\delta+1) / 3$ of the first and last, respectively, copy of $K_{\delta}$. Let $\mathcal{S}$ be the 
set of all k-element sets of vertices of $G_{d, \delta}$ that are contained in $\bigcup_{i=0}^{d} V_{i}$, and that have no two vertices in the same $V_{i}$. Let $F$ be the path of order $d+1$ with vertices $u_{0}, u_{1}, \ldots, u_{d}$. We define a mapping $f$ that maps every set in $\mathcal{S}$ to a $k$-set of vertices of $F$. For $S \in \mathcal{S}$ let $f(S)$ be the subset of $V(F)$ containing those $u_{i}$ for which $S$ contains a vertex in $V_{i}$. It is clear that $d_{G_{d, \delta}}(S)=d_{F}(f(S))$. Since every $k$-set of vertices of $F$ is the image under $f$ of exactly $\left(\frac{\delta+1}{3}\right)^{k}$ sets in $\mathcal{S}$, we have

$$
S W_{k}\left(G_{d, \delta}\right) \geq \sum_{S \in \mathcal{S}} d_{G_{d, \delta}}(S)=\sum_{S \in \mathcal{S}} d_{P_{d+1}}(f(S))=\sum_{S \subseteq V(F),|S|=k}\left(\frac{\delta+1}{3}\right)^{k} d_{F}(S) .
$$

Hence, by Theorem 1,

$$
S W_{k}\left(G_{d, \delta}\right) \geq\left(\frac{\delta+1}{3}\right)^{k} S W_{k}(F)=\left(\frac{\delta+1}{3}\right)^{k} \frac{(k-1)(d+2)}{k+1}\left(\begin{array}{c}
d+1 \\
k
\end{array}\right) .
$$

Now for constant $k$ and $\delta$ and large $n$ and $d$ we get $n=d \frac{\delta+1}{3}+O(1)$ and thus $d=\frac{3 n}{\delta+1}+O(1)$. Hence $\left(\begin{array}{c}d+1 \\ k\end{array}\right)=\left(\frac{3}{\delta+1}\right)^{k}\left(\begin{array}{l}n \\ k\end{array}\right)+O\left(n^{k-1}\right)$, and so

$$
\begin{aligned}
S W_{k}\left(G_{d, \delta}\right) & \geq\left(\frac{\delta+1}{3}\right)^{k} \frac{(k-1)(d+2)}{k+1}\left[\left(\frac{3}{\delta+1}\right)^{k}\left(\begin{array}{l}
n \\
k
\end{array}\right)+O\left(n^{k-1}\right)\right] \\
& =\frac{k-1}{k+1} \frac{3 n}{\delta+1}\left(\begin{array}{l}
n \\
k
\end{array}\right)+O\left(n^{k}\right) .
\end{aligned}
$$

Dividing by $\left(\begin{array}{l}n \\ k\end{array}\right)$ we get

$$
\mu_{k}\left(G_{d, \delta}\right)=\frac{k-1}{k+1} \frac{3 n}{\delta+1}+O(1)
$$

as desired.

\section{An improved bound for triangle-free graphs}

Our main aim in this section is to improve the bound in Theorem 3 for triangle-free graphs. The basic idea of the proof of the improved bound is similar to Theorem 3 , but some additional arguments are needed.

For an edge $e=u v$ of a triangle-free graph $G$ denote by $T(e)$ the subtree of $G$ whose vertex set is $N(u) \cup N(v)$, and whose edges are the edges joining $u$ or $v$ to its neighbours in $G$. The distance $d\left(e_{1}, e_{2}\right)$ between two edges of $G$ is the minimum of the four distances between a vertex incident with $e_{1}$ and a vertex incident with $e_{2}$. The distance between an edge $e$ and a set $E_{1}$ of edges is the minimum of the distances between $e$ and the edges in $E_{1}$.

Lemma 3. Let $T$ be a tree and $L$ the line graph of $T$. Let $S_{V}$ be a set of vertices of $T$, and $S_{E}$ a set of edges of $T$ such that $S_{V} \subseteq V\left(S_{E}\right)$. Then

$$
d_{T}\left(S_{V}\right) \leq d_{L}\left(S_{E}\right)+1
$$


Proof: Let $T_{L}\left(S_{E}\right)$ be a Steiner tree for $S_{E}$ in $L$ and let $U$ be its vertex set. Then $U$ is a set of $d_{L}\left(S_{E}\right)+1$ vertices of $L$. Since $U \subseteq E(T)$ and since $U$ induces a connected graph in $L$, the subgraph $T[U]$ of $T$ induced by the set of edges $U$ is also connected. Since $T[U]$ contains all vertices of $S_{V}$, we have $d_{T}\left(S_{V}\right) \leq|U|=$ $d_{L}\left(S_{E}\right)+1$, as desired.

Theorem 4. Let $G$ be a connected, triangle-free graph of order $n$ with minimum degree $\delta$. Then $G$ contains a spanning tree $T$ with

$$
S W_{k}(T) \leq \frac{k-1}{k+1} \frac{2(n+1)}{\delta}\left(\begin{array}{l}
n \\
k
\end{array}\right)+\left(\frac{4 \delta-2}{\delta}+3 k+1\right)\left(\begin{array}{l}
n \\
k
\end{array}\right) .
$$

Proof: To construct a matching of $G$ start by choosing an edge $b_{1}$ and letting $M_{1}=\left\{b_{1}\right\}$ and $T_{1}=T\left(b_{1}\right)$. Let $b_{2}$ be an edge at distance exactly 3 from $M_{1}$, if one exists. Then there exists an edge $e_{2}$ joining some vertex of $T_{1}$ to some vertex of $T\left(b_{2}\right)$. Let $M_{2}=M_{1} \cup\left\{b_{2}\right\}$ and let $T_{2}$ be the tree obtained from $T_{1} \cup T\left(b_{2}\right)$ by adding the edge $e_{2}$. Let $b_{3}$ be an edge at distance exactly 3 from $M_{2}$, if one exists. Then there exists an edge $e_{3}$ joining some vertex of $T_{2}$ to some vertex of $T\left(b_{3}\right)$. Let $M_{3}=M_{2} \cup\left\{b_{3}\right\}$ and let $T_{3}$ be the graph obtained from $T_{2} \cup T\left(b_{3}\right)$ by adding the edge $e_{3}$. Generally, given $M_{i}$ and $T_{i}$, we choose an edge $b_{i+1}$ at distance exactly 3 from $M_{i}$, if one exists, let $e_{i+1}$ be an edge joining a vertex in $T_{i}$ to a vertex in $T\left(b_{i+1}\right)$, let $M_{i+1}=M_{i} \cup\left\{b_{i+1}\right\}$, and let $T_{i+1}$ be the tree obtained from $T_{i} \cup T\left(b_{i+1}\right)$ by adding the edge $e_{i+1}$. Repeat this step until, after $s$ steps say, all edges are at distance at most 2 from $M_{s}$. Let $T_{0}:=T_{s}$ and $M:=M_{s}$. Then $M$ is a matching and every edge of $G$ is within distance at most 2 in $G$ from $M$, and so every vertex of $G$ is at distance at most three from $V(M)$. Joining every vertex not in $T_{0}$ to a neighbour that is closer to $T_{0}$ or in $T_{0}$ yields a spanning tree $T$ of $G$ that preserves the distances from all vertices to $V(M)$.

We now show that $S W_{k}(T)$ is bounded as claimed. For every vertex $v$ of $T$ choose a nearest vertex $a_{v} \in V(M)$, and a shortest $\left(v, a_{v}\right)$-path $P_{v}$ in $T$. Then $P_{v}$ has at most three edges. For $a \in V(M)$ let $c(a)$ be the number of vertices $v$ of $G$ with $a_{v}=a$. Since $M$ is a matching and since $G$ is triangle-free, for every $a \in V(M)$ all vertices in $N_{G}[a]$ except the matching partner of $a$ have $a$ as their nearest vertex in $M$, and so

$$
c(a) \geq \delta \text { for all } a \in M,
$$

Making use of the fact that every vertex of $T$ is within distance three of some vertex in $V(M)$, we show as in the proof of Theorem 3 (see equation (4) there) we show

$$
S W_{k}(T) \leq S W_{k}(T, c)+3 k\left(\begin{array}{l}
n \\
k
\end{array}\right) .
$$

Let $L(T)$ be the line graph of $T$. Define a weight function $c_{2}$ on the vertices of $L(T)$, i.e., the edges of $T$, by

$$
c_{2}(u v)=\left\{\begin{array}{cl}
c(u)+c(v) & \text { if } u v \in M \\
0 & \text { if } u v \notin M .
\end{array}\right.
$$


Clearly, $c(V(T))=c_{2}(M)=n$. We now show that

$$
S W_{k}(T, c) \leq S W_{k}\left(L, c_{2}\right)+\left(\begin{array}{l}
n \\
k
\end{array}\right) .
$$

Define a bijection $f: V(T)_{c} \rightarrow V(L)_{c_{2}}$ that maps, for every edge $u v \in M,\left\{u^{1}, u^{2} \ldots, u^{c(u)}\right\} \cup$ $\left\{v^{1}, v^{2} \ldots, v^{c(u)}\right\}$ to $\left\{u v^{1}, u v^{2} \ldots, u v^{c_{2}(u v)}\right\}$. If $S \subseteq V_{c}$ then $f(S) \subseteq E(T)_{c_{2}}$ and clearly $S^{*} \subseteq V\left(f(S)^{*}\right)$. Hence, by Lemmal 3 this implies

$$
d_{T}\left(S^{*}\right) \leq d_{L}\left(f(S)^{*}\right)+1
$$

and so

$$
d_{T}(S) \leq d_{L}(f(S))+1
$$

Summation over all $k$-element subsets $S$ of $V_{c}$ yields

$$
\begin{aligned}
\sum_{S \subseteq V_{c},|S|=k} d_{T}(S) & \leq \sum_{S \subseteq V_{c},|S|=k}\left(d_{L}(f(S))+1\right) \\
& =\sum_{S \subseteq E(T)_{c_{2}},|S|=k}\left(d_{L}(f(S))+1\right) \\
& =S W_{k}\left(L, c_{2}\right)+\left(\begin{array}{l}
n \\
k
\end{array}\right),
\end{aligned}
$$

which is (10).

By the construction of $T_{0}$ and $T$ every edge $b_{i}, i>1$, is at distance exactly three in $T$ from some edge $b_{j}$ with $j<i$. It follows that in the line graph $L$ every vertex $b_{i}$ of $L$ with $i>1$ is at distance exactly four from some vertex $b_{j}$ with $j<i$. Therefore,

$$
L^{4}[M] \text { is connected. }
$$

Let $H=L^{4}[M]$. Since $d_{L}\left(b_{i}, b_{j}\right) \leq 4 d_{H}\left(b_{i}, b_{j}\right)$ for all $b_{i}, b_{j} \in M$, and since the weight of $c_{2}$ is concentrated in $M$, we have

$$
S W_{k}\left(L, c_{2}\right) \leq 4 S W_{k}\left(H, c^{\prime}\right)
$$

where $c^{\prime}$ is the restriction of $c$ to $M$. By (8) $c^{\prime}\left(b_{i}\right) \geq 2 \delta$ for all $b_{i} \in M$. Moreover, the total weight of $c^{\prime}$ is $n$, i.e., $N=n$. Hence, by Lemma 2

$$
S W_{k}\left(H, c^{\prime}\right) \leq \frac{k-1}{k+1} \frac{n+1}{2 \delta}\left(\begin{array}{l}
n \\
k
\end{array}\right)+\frac{2 \delta-1}{2 \delta}\left(\begin{array}{l}
n \\
k
\end{array}\right) .
$$

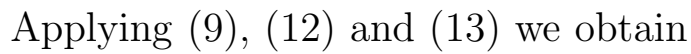

$$
S W_{k}(T) \leq \frac{k-1}{k+1} \frac{2(n+1)}{\delta}\left(\begin{array}{l}
n \\
k
\end{array}\right)+\left(\frac{4 \delta-2}{\delta}+3 k+1\right)\left(\begin{array}{l}
n \\
k
\end{array}\right),
$$

as desired. 
Corollary 2. Let $G$ be a connected graph of order $n$ with minimum degree $\delta$. Then

$$
S W_{k}(G) \leq \frac{k-1}{k+1} \frac{2(n+1)}{\delta}\left(\begin{array}{l}
n \\
k
\end{array}\right)+\left(\frac{4 \delta-2}{\delta}+3 k+1\right)\left(\begin{array}{l}
n \\
k
\end{array}\right),
$$

and thus

$$
\mu_{k}(G) \leq \frac{k-1}{k+1} \frac{2(n+1)}{\delta}+\frac{4 \delta-2}{\delta}+3 k+1 .
$$

Example 2. The following example shows that the bound on the Steiner $k$-Wiener index in Corollary 2 is best possible apart from a term $O\left(n^{k}\right)$, and that the bound on the average Steiner $k$-distance is best possible apart from an additive constant. We only construct examples for the case that $\delta$ is even, but as in Example 1 it is not difficult to modify this construction for odd values of $\delta$. For $d \in \mathbb{N}$ define the graph $H_{d, \delta}$ by

$$
H_{d, \delta}=\delta K_{1}+\delta K_{1}+K_{\delta / 2}+K_{\delta / 2}+\cdots+K_{\delta / 2}+K_{\delta / 2}+\delta K_{1}+\delta K_{1},
$$

where the term $K_{\delta / 2}$ appears $d-3$ times. Then calculations similar to those in Example 1 show that for constant $\delta$ and $k$ and large $n$ and $d$ we have

$$
S W_{k}\left(H_{d, \delta}\right) \leq \frac{k-1}{k+1} \frac{2(n+1)}{\delta}\left(\begin{array}{l}
n \\
k
\end{array}\right)+O\left(n^{k}\right),
$$

and thus

$$
\mu_{k}\left(H_{d, \delta}\right) \leq \frac{k-1}{k+1} \frac{2 n}{\delta}+O(1)
$$

\section{References}

[1] Ali, P.; Dankelmann, P.; Mukwembi, S.; Upper bounds on the Steiner diameter of a graph. Discrete Appl. Math. 160 issue 12 (2012), 1845-1850.

[2] Ali, P.; The Steiner diameter of a graph with prescribed girth. Discrete Math. 313 no. 12 (2013), 1322-1326.

[3] Ali, P.; Dankelmann, P.; Mukwembi, S.; The Steiner diameter of 3-, 4-, and 5-connected maximal planar graphs. Discrete Appl. Math. 179 (2014), 222-228.

[4] Beezer, R.A.; Riegsecker, J.E.; Smith, B.A.; Using minimum degree to bound average distance. Discrete Math. 226 no. 1-3 (2001), 365-371.

[5] Chartrand, G.; Oellermann, O.R.; Tian, S.; Zou, H.B.; Steiner distance in graphs, Casopis Pest. Mat. 114 (1989) 399-410.

[6] Dankelmann, P.; Oellermann, O.R.; Swart, H.C.; The average Steiner distance of a graph, J. Graph Theory, 22 (1996), 15-22. 
[7] Dankelmann, P.; Oellermann, O.R.; Swart, H.C.; Bounds on the Steiner diameter of a graph. Proceedings of the 8th Quadrennial Conference on Graphs, Combinatorics, Algorithms and its Applications at Western Michigan University, Kalamazoo (Michigan, USA), 1996, Vol. 1 (1998), 269-279.

[8] Dankelmann, P.; Oellermann, O.R.; Swart, H.C.; On the average Steiner distance of certain classes of graphs. Discrete Appl. Math, 79 (1997), 91-103.

[9] Dankelmann, P.; Entringer, R.; Average distance, minimum degree and spanning trees. J. Graph Theory 33 no 1 (2000), 1-13.

[10] Dankelmann, P.; Mukwembi, S.; Swart, H.C.; Average distance and edgeconnectivity II. SIAM J. Discrete Math. 21 (2008), 1035-1052.

[11] Dankelmann, P.; Mukwembi, S.; Swart, H.C.; Average distance and edgeconnectivity I. SIAM J. Discrete Math. 22 (2008), 92-101.

[12] Dankelmann, P.; Mukwembi, S.; Swart, H.C.; Average distance and vertex connectivity. J. Graph Theory 62 (2009), 157-177.

[13] Das, K.Ch.; Nadjafi-Arani, M.J.; On maximum Wiener index of trees and graphs with given radius. J. Combin. Optim. 34 (2017), 574-587.

[14] Doyle, J.K.; Graver, J.E.; Mean distance in a graph. Discrete Math. 7 (1977), $147-154$.

[15] Entringer, R.C.; Jackson, D.E.; Snyder, D.A.; Distance in graphs. Czech Math. J. 26 (1976), 283-296.

[16] Fajtlowicz, S.; Waller, W.A.; On conjectures of GRAFFITI II. Congr. Numer. 60 (1987), 187-197.

[17] Klavžar, S.; Nadjafi-Arani, M.J.; Wiener index in weighted graphs via unification of $\theta^{*}$-classes. European J. Combin. 36 (2014), 71-76.

[18] Knor, M.; Luzar, B.; Škrekovski, R.; Gutman, I.; On Wiener index of common neighborhood graphs. MATCH Commun. Math. Comput. Chem. 72 no. 1 (2014), 321-332.

[19] Knor, M.; Škrekovski, R.; Tepeh, A.; Mathematical aspects of Wiener index. Ars Mathematica Contemporanea, 11 no. 2 (2016), 327-352.

[20] Kouider, M.; Winkler, P.; Mean distance and minimum degree. J. Graph Theory 25 no. 1 (1997): 95-99.

[21] Li, X.; Mao, Y.; Gutman, I.; The Steiner Wiener index of a graph. Discuss. Math. Graph Theory 32 no. 2 (2016), 455-465. 
[22] Li, X.; Mao, Y.; Gutman, I.; Inverse problem on the Steiner Wiener index. Discuss. Math. Graph Theory 38 no. 1 (2018), 83-95.

[23] Lovász, L.; Combinatorial Problems and Exercises. Akadémiai Kiadó (Budapest), 1979.

[24] Lu, L.; Huang, Q.; Hou, J.; Chen, X.; A sharp lower bound on the Steiner Wiener index for trees with given diameter. Discrete Math. 341 no. 3 (2018), 723-731.

[25] Mao, Y.; The Steiner diameter of a graph. arXiv preprint arXiv:1509.02801.

[26] Mao, Y.; Wang, Z.; Gutman, I.; Steiner Wiener index of graph products. Transactions on Combinatorics 5 no. 3 (2016), 39-50.

[27] Mao, Y.; Wang, Z.; Gutman, I.; Li, H.; Nordhaus-Gaddum type results for the Steiner Wiener index of graphs. Discrete Appl. Math, 219 (2017), 167-175.

[28] Mukwembi, S.; Vetrík, T.; Wiener index of trees of given order and diameter at most 6. Bull. Austral. Math. Soc. 89 (2014), 379-396.

[29] Plesník, J.; On the sum of all distances in a graph or digraph. J. Graph Theory 8 (1984), 1-24.

[30] Rouvray, D.H.; The rich legacy of half century of the Wiener index. In: Topology in Chemistry - Discrete Mathematics of Molecules, D.H. Rouvray and R.B. King (eds.), Horwood, Chichester (2002) 16-37.

[31] Wagner, S.; A class of trees and its Wiener index. Acta Appl. Math. 91 no. 2 (2006), 119-132.

[32] Wang, H.; Yu, G.; All but 49 numbers are Wiener indices of trees. Acta Appl. Math. 91 no. 2 (2006), 15-20.

[33] Wiener, H. Structural determination of paraffin boiling points. J. Amer. Chem. Soc. 69 (1947), 17-20.

[34] Yeh, Y.-N.; Gutman, I.; On the sum of all distances in composite graphs. Discrete Math. 134 (1994), 359-365.

[35] Zhang, L.; Wu, B.; The Nordhaus-Gaddum type inequalities for some chemical indices. MATCH Commun. Math. Comput. Chem. 54 (2005), 189-194. 